\title{
Cystic Lymphangioma of the Abdominal Wall in Childhood: A Report of Two Cases Focused on Radiologic Findings
}

\author{
In Kyeom Kim, ${ }^{1}$ and Jung Wook Seo ${ }^{1, *}$ \\ ${ }^{1}$ Department of Radiology, Ilsan Paik Hospital, Inje University College of Medicine, Goyang-Si, Gyeonggi-Do, Republic of Korea \\ "Corresponding author: Jung Wook Seo, Department of Radiology, Ilsan Paik Hospital, Inje University College of Medicine, Goyang-Si, Gyeonggi-Do, Republic of Korea. Tel: \\ +82-319107389, Fax: +82-319107369, E-mail: seojwrad@paik.ac.kr
}

Received 2016 July 02; Revised 2016 October 14; Accepted 2016 November 26.

\begin{abstract}
A cystic lymphangioma is a rare benign tumor of the lymphatic tissue that mostly affects the head and neck, and usually manifests during childhood. Cystic lymphangioma of the abdominal wall is extremely rare. Herein, we report two young children with cystic lymphangioma of the abdominal wall. In one of the cases, the properitoneal fat layer of the abdominal wall was affected. We hereby analyze radiologic features of cystic lymphangioma of the abdominal wall.
\end{abstract}

Keywords: Abdominal Wall, Child, Lymphangioma, Cystic

\section{Introduction}

Cystic lymphangioma is a rare congenital malformation of the lymphatic system resulting from sequestered primitive lymphatic tissue that prevents communication between a lymphatic sac and the venous system. It has been reported to account for $5 \%$ - $6 \%$ of all pediatric neoplasms (1). Few reports on cases of cystic lymphangioma of the abdominal wall have been published previously. Herein, we present the clinical and radiological findings of two young children with cystic lymphangioma of the abdominal wall.

\section{Case Presentation}

\subsection{Case 1}

A one-year-old girl presented with a painless mass that was accidentally discovered by her parents in the left lower abdomen. Physical examination revealed a palpable soft mass $6 \mathrm{~cm}$ in diameter in the left lower abdominal wall. All laboratory test results were within normal limits.

Initial abdominal ultrasonography(US) showed a large mass with a maximum diameter of $7 \mathrm{~cm}$. The mass included a well-defined cystic lesion divided by thin internal septa (Figure 1A). Contrast-enhanced abdominopelvic computed tomography (CT) was performed to further assess the mass, and it showed a well-defined, lobulated cystic mass measuring $6.6 \times 4.1 \times 4.8 \mathrm{~cm}$ in the left pelvic cavity. Additionally, the wall of the mass was uniformly thick (Figure 1B-D). The mass displaced the urinary bladder inferolaterally and the small bowel superiorly. It extended to the subcutaneous layer of the left lower abdominal wall; however, there was no destruction of the layers of the abdominal wall. Moreover, CT scan demonstrated that the mass was surrounded by prominent properitoneal fat and that it elevated the adjacent peritoneal lining. The contents of the mass were homogeneous in density, without enhancement. Based on these findings, our primary diagnosis was cystic lymphangioma originating from the abdominal wall. We considered other cystic masses, such as ovarian cystic neoplasms and cystic mesotheliomas in the differential diagnosis.

A peritoneal incision was made to assess the mass. The fluid-containing mass was located in the subcutaneous and muscle layers of the abdominal wall, and it bulged into the extraperitoneal pelvic cavity without invading the peritoneal cavity. Surgical excision was eventually performed.

Histopathological examination revealed a cystic lymphangioma arising from the abdominal wall in the left lower quadrant (LLQ) and extending to the properitoneal fat layer (Figure 1E).

\subsection{Case 2}

A 4-year-old boy presented with a mass that was progressively growing in the right upper quadrant (RUQ) of the abdomen since infancy. Physical examination revealed a soft compressible mass in the RUQ of the abdomen.

Abdominal US showed a well-defined, lobulated cystic mass lesion measuring $6.8 \times 1.7 \times 11.7 \mathrm{~cm}$, with many thin septa. Color Doppler US showed no blood flow (Figure 2A). Abdominal magnetic resonance imaging (MRI) was performed to further assess the mass. It showed a multiloculated cystic lesion measuring $7.3 \times 2.5 \times 10.3 \mathrm{~cm}$ in 

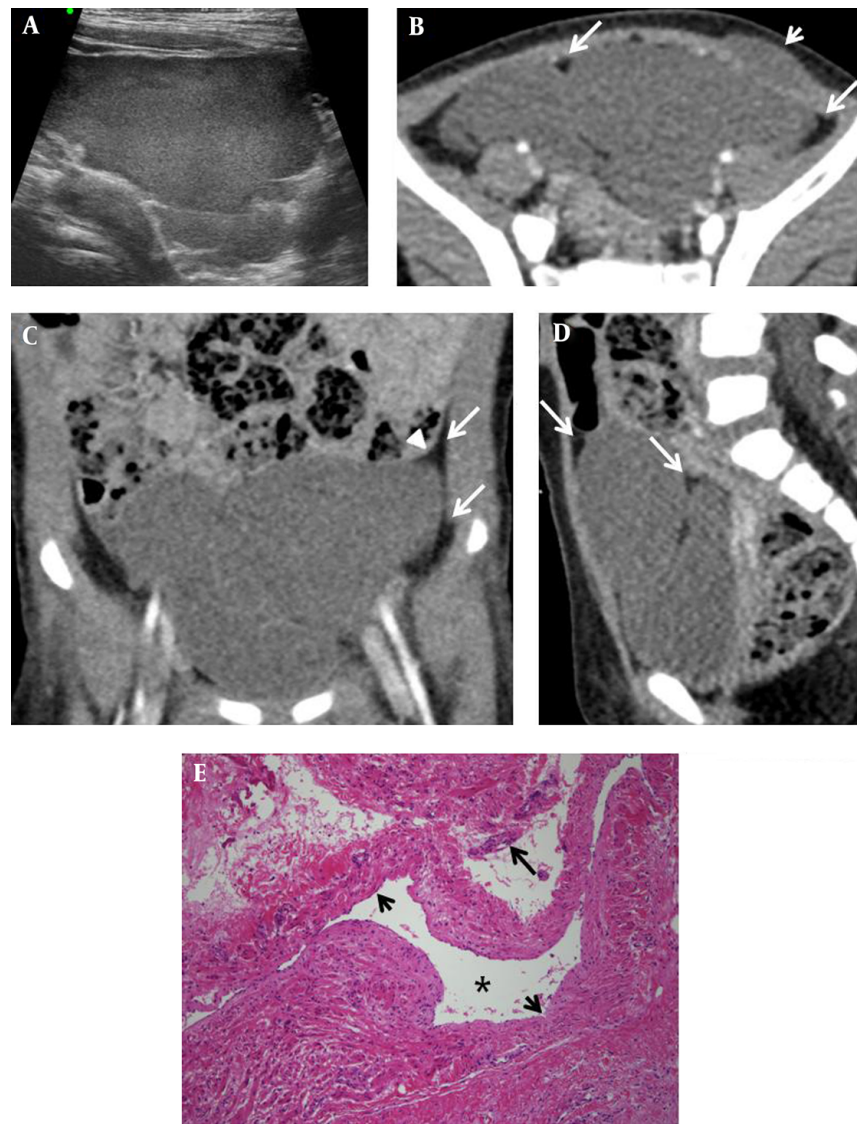

Figure 1. A one-year-old girl with cystic lymphangioma of the abdominal wall; A, Abdominal ultrasonography image shows a lobulated cystic tumor in the left lower abdomen. $\mathrm{B}$, Contrast-enhanced abdominopelvic computed tomography axial image shows a lobulated, homogeneous mass in the pelvic cavity, which extends to the subcutaneous layer of the left lower abdominal wall, without destruction of the abdominal wall (short arrow). The mass is surrounded by prominent properitoneal fat (long arrows), and it displaces the urinary bladder to the right. C, Elevated properitoneal lining (arrowhead) and prominent properitoneal fat (long arrows) are seen in the coronal view. D, Sagittal image shows that the mass is surrounded by prominent properitoneal fat (long arrows), and it displaces the urinary bladder inferiorly and the small bowel superiorly. E, Photomicrograph of the excised mass shows dilated lymphatic spaces (asterisk) lined by a flattened layer of endothelial cells (short arrows). The walls of the cyst show scattered lymphoid cells (long arrow) (hematoxylin and eosin staining, $\times 100$ ).

the subcutaneous and muscle layers of the right anterolateral trunk. Additionally, low signal intensity was noted in T1-weighted images (T1WI) and bright high signal intensity was noted in T2-weighted images (T2WI). The mass extended from the right upper abdominal wall to the right lower chest wall (Figure 2B, C). MRI findings were compatible with a cystic lymphangioma.

The patient underwent surgery for mass removal, and intraoperatively, the multiloculated cystic mass was located in the subcutaneous and muscle layers of the anterior abdominal wall in the RUQ. The mass was eventually excised. Histopathological examination of the specimen showed large, thin-walled lymphatic channels in loose connective tissue stroma (Figure 2D). All findings were compatible with the diagnosis of cystic lymphangioma.

\section{Discussion}

The majority of cystic lymphangiomas (90\%) develop during the first 2 years of life, and $65 \%$ are noted at birth. Prenatal diagnosis is made by US during the nuchal translucency test (1). It has been reported that $75 \%$ of cases involve the neck, $20 \%$ involve the axilla, and the remaining $5 \%$ involve various body parts, including retroperitoneum, mediastinum, pelvis, and groin (2).

Hancock et al. (3) reported the following anatomical distributions of cystic lymphangiomas: cervical, $31.4 \%$; craniofacial, $18.9 \%$; extremities, $18.9 \%$; trunk, 9.2\%; intraabdominal, 9.2\%; cervicoaxillothoracic, $4.9 \%$; multiple, $3.8 \%$; cervicomediastinal, $2.2 \%$; and intrathoracic, $1.6 \%$. Pandit et al. (4) reviewed 45 cases of cystic lymphangioma in children and noted cystic lymphangioma of the abdominal wall in only two of these cases (4.4\%). 

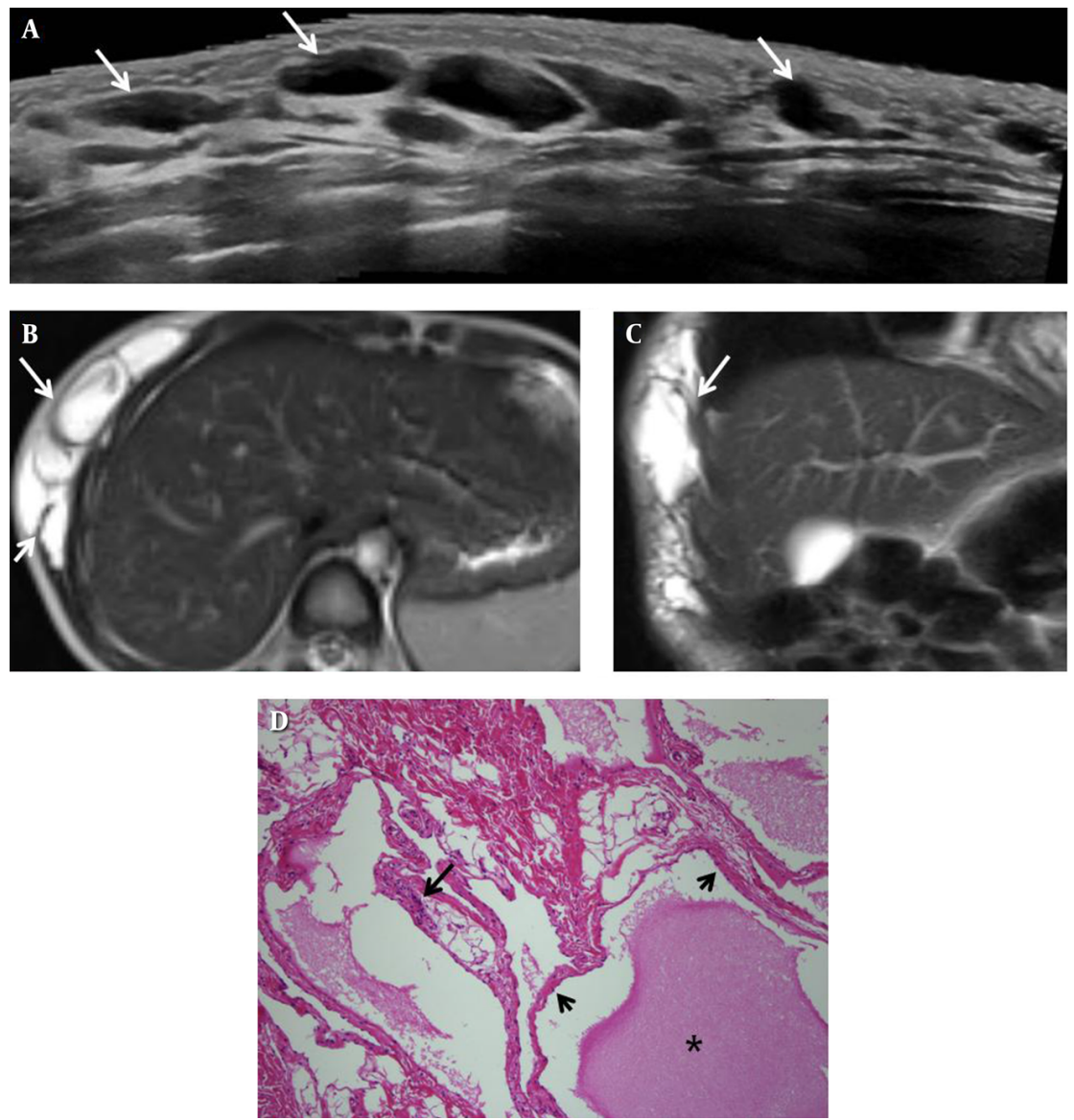

Figure 2. A 4-year-old boy with a cystic lymphangioma of the abdominal wall. A, Abdominal ultrasonography image shows a multiloculated cystic mass (long arrow) in the subcutaneous layer of the right upper abdominal wall. B, T2-weighted magnetic resonance axial image of the abdomen shows a multiloculated cystic lesion (long arrow) in the subcutaneous layer of the right anterolateral trunk and intramuscular extension (short arrow). C, Coronal image shows that the multiloculated cystic mass (long arrow) extends into the right lower chest wall; D, Photomicrograph of the excised specimen shows dilated lymphatic spaces filled with homogeneous eosinophilic and proteinaceous material (asterisk), and lined by a flattened layer of endothelial cells (short arrows); The walls of the cyst show scattered lymphoid cells (long arrow) (hematoxylin and eosin staining, $\times 100)$.

To our knowledge, less than 4 cases of cystic lymphangioma of the abdominal wall have been published in the English literature. We performed a PubMed search and found two relevant articles published between 2003 and $2015(5,6)$. These two articles presented a total of two cases of cystic lymphangioma of the abdominal wall and provided radiological images. Therefore, there are a total of four cases with radiological images, including our cases.

In our first case, a lobulated cystic mass was found in the abdominal wall in the LLQ, and the mass was bulging into the properitoneal fat layer. This bulging pelvic mass was surrounded by prominent properitoneal fat, and it appeared to elevate the adjacent peritoneal lining. Thus, our primary diagnosis was cystic lymphangioma in the pelvic extraperitoneal space. In our second patient, the mass was confined to the anterior muscular and subcutaneous lay- ers of the RUQ, and there was no bulging into the properitoneal fat layer.

Ammar et al. (5) reported a case of a 10-year-old girl who complained of swelling on the left side of the abdomen caused by a cystic lymphangioma. The mass predominantly bulged into the pelvic cavity without subcutaneous layer involvement. However, it was not possible to evaluate the relationship between the cystic mass and the peritoneal lining because of the poor quality of the single available image.

Riahinezhad et al. (6) reported a case of a 9-year-old boy with sudden onset of localized abdominal pain and bulging caused by rapid expansion of a hemorrhagic cystic lymphangioma of the abdominal wall. The right subphrenic mass appeared compress the anterior part of the liver, and it was surrounded by properitoneal fat. There 
was no involvement of the subcutaneous layer.

Almost all the above-mentioned cases shared similar characteristic radiological findings. US generally demonstrated a multiloculated cystic mass with thin internal septa, and color Doppler US showed absence of blood flow. The cyst was either anechoic or contained echogenic debris. CT showed a large, thin-walled, multiseptated cystic mass advancing into surrounding organs. MRI showed high signal intensity in T2WIs and low signal intensity in T1WIs.

In three of the four cases, including our cases, the cystic lymphangioma involved the properitoneal fat layer, and the mass bulged into the extraperitoneal pelvic cavity or the peritoneal cavity. Therefore, it is important to differentiate between a cystic lymphangioma involving the properitoneal fat layer and other cystic masses in the peritoneal cavity. The presence of peritoneal lining elevation and prominent properitoneal fat surrounding the mass can help in determining whether the mass is located within the extraperitoneal pelvic cavity. Attention to these signs is essential for an accurate radiological diagnosis of cystic lymphangioma of the abdominal wall. On the other hand, a cystic lymphangioma involving the subcutaneous layer tends to be discovered early because of the patient's clinical symptoms.

The treatment of a cystic lymphangioma remains challenging. Surgical excision has been considered as the standard treatment. However, the results are often unsatisfactory because of complications including recurrence due to incomplete excision, damage to surrounding blood vessels and nerves, and infection (7). Alternative treatments, such as use of sclerosing agents, laser therapy, radiation therapy and chemotherapy have been reported in patients who are poor surgical candidates. The recurrence rate is low if the tumor is completely excised. However, it may be difficult to obtain a safe surgical margin because of the tendency of the lesion to infiltrate surrounding tissues. A previous report mentioned that percutaneous sclerosis or a combination of percutaneous sclerosis and surgical excision was successful (8).

In conclusion, we presented two rare cases of cystic lymphangioma of the abdominal wall that were treated with surgical excision. Additionally, we summarized the characteristic imaging findings and extent of involvement in these cases. We suggest that the diagnostic imaging protocol in patients suspected with cystic lymphangioma should include US examination initially and MRI with contrast administration to further investigate the lesion features in order to evaluate the presence of malignancy.

\section{Acknowledgments}

None declared.

\section{Footnotes}

Authors' Contributions: Kyeom Kim drafted and edited the manuscript, and performed a literature search. Jung Wook Seo designed and edited the manuscript, and provided final approval.

Financial Disclosure: None declared.

Funding/Support: None declared.

\section{References}

1. Faul JL, Berry GJ, Colby TV, Ruoss SJ, Walter MB, Rosen GD, et al. Thoracic lymphangiomas, lymphangiectasis, lymphangiomatosis, and lymphatic dysplasia syndrome. Am J Respir Crit Care Med. 2000;161(3 Pt 1):1037-46. doi: 10.1164/ajrccm.161.3.9904056. [PubMed: 10712360].

2. Malnofski MJ, Poulton TB, Nazinitsky KJ, Hissong SL. Prenatal ultrasonic diagnosis of retroperitoneal cystic lymphangioma. J Ultrasound Med. 1993;12(7):427-9. [PubMed: 8355339].

3. Hancock BJ, St-Vil D, Luks FI, Di Lorenzo M, Blanchard H. Complications of lymphangiomas in children. J Pediatr Surg. 1992;27(2):220-4. doi: 10.1016/0022-3468(92)90316-Y. [PubMed:1564622].

4. Pandit SK, Rattan KN, Budhiraja S, Solanki RS. Cystic lymphangioma with special reference to rare sites. Indian J Pediatr. 2000;67(5):339-41. doi: 10.1007/BF02820682. [PubMed:10885205].

5. Ammar S, Jallouli M, Ben Dhaou M, Mhiri R. Cystic lymphangioma of the rectus abdominis muscle: an extremely rare clinical entity. Iran J Pediatr. 2014;24(5):662-3. [PubMed: 25793081].

6. Riahinezhad M, Sarrami AH, Shariat Z, Taghizadeh F. Two unusual sites of cystic lymphangioma in a child: A report of imaging profile with surgical and histopathologic findings. Adv Biomed Res. 2015;4:169. doi: 10.4103/2277-9175.162546. [PubMed: 26436083].

7. Tran Ngoc N, Tran Xuan N. Cystic hygroma in children: a report of 126 cases.JPediatr Surg. 1974;9(2):191-5. doi: 10.1016/S0022-3468(74)80120-X. [PubMed: 4825790].

8. Okazaki T, Iwatani S, Yanai T, Kobayashi H, Kato Y, Marusasa T, et al. Treatment of lymphangioma in children: our experience of 128 cases. J Pediatr Surg. 2007;42(2):386-9. doi: 10.1016/j.jpedsurg.2006.10.012. [PubMed: 17270554]. 